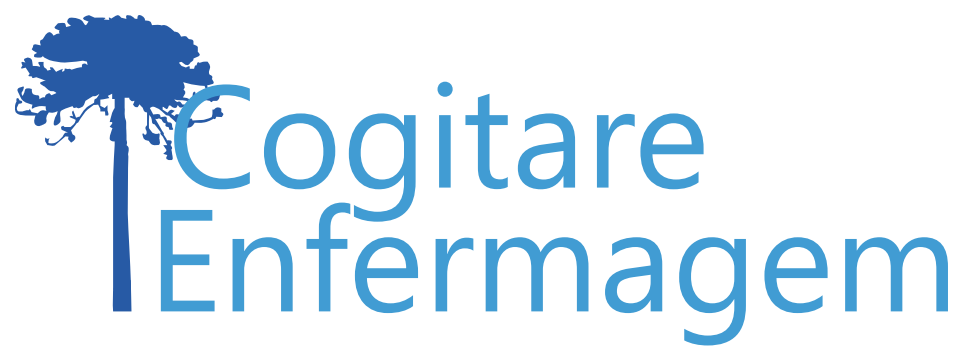

\title{
AVALIAÇÃO DE TREINAMENTO SOBRE PRIMEIROS SOCORROS PARA EQUIPE TÉCNICA DE ESCOLAS DE ENSINO ESPECIALIZADO
}

Jackeline Gonçalves Brito1, Ingrydy Maria da Silva², Christine Baccarat de Godoy³ Ana Paula dos Santos Jesus Marques França ${ }^{4}$

\section{RESUMO}

Objetivo: analisar o efeito de atividade educativa sobre primeiros socorros em acidentes escolares. Metodologia: estudo quase experimental do tipo antes e depois, realizado com a equipe técnica de escolas de ensino especializado para pessoas com deficiência, no estado do Mato Grosso, entre fevereiro e agosto de 2017. Foi realizado o teste não paramétrico de McNemar para analisar o efeito da atividade educativa.

Resultados: participaram 76 profissionais de nível médio, $62(81,6 \%)$ eram do sexo feminino, $66(86,8 \%)$ eram Auxiliares de Desenvolvimento Infantil. Dos profissionais, 35 (46,1\%) nunca participaram de treinamento ou atividade educativa sobre primeiros socorros. Houve significância para o aumento de acerto em todas as questões após o treinamento $(p<0,05)$.

Conclusão: os profissionais da equipe técnica apresentaram conhecimento prévio insuficiente sobre primeiros socorros e o treinamento melhora a segurança dos alunos e contribui para a sociedade em que estão inseridos. Evidencia-se a importância da inserção do enfermeiro no ambiente escolar.

DESCRITORES: Primeiros socorros; Capacitação; Pessoas com deficiência; Educação especial; Crianças.

COMO REFERENCIAR ESTE ARTIGO:

Brito JG, Silva IM da, Godoy CB de, França AP dos SJM. Avaliação de treinamento sobre primeiros socorros para equipe técnica de escolas de ensino especializado. Cogitare enferm. [Internet]. 2019 [acesso em "colocar data de acesso, dia, mês abreviado e ano"]; 24. Disponível em: http://dx.doi.org/10.5380/ce.v24i0.60340.

Este obra está licenciado com uma Licença Creative Commons Atribuiçãa 4.0 Internacional.

${ }^{1}$ Enfermeira. Doutoranda em Enfermagem. Universidade Federal de Mato Grosso. Cuiabá, MT, Brasil. () ${ }^{2}$ Discente de Enfermagem. Universidade Federal de Mato Grosso. Cuiabá, MT, Brasil. $\odot$

${ }^{3}$ Enfermeira. Pós-Doutora em Saúde Pública. Docente de Enfermagem da Universidade Federal de Mato Grosso. Cuiabá, MT, Brasil. 9

${ }^{4}$ Enfermeira. Doutora em Ciências Filosóficas. Professora Coordenadora da Escola Superior de Enfermagem do Porto. Porto, Portugal. () 


\title{
EVALUATION OF FIRST AID TRAINING FOR THE TECHNICAL STAFF OF SPECIAL EDUCATION SCHOOLS
}

\begin{abstract}
Objective: to analyze the effect of an educational activity on first aid in school accidents.

Methodology: a quasi-experimental, before-and-after type study, carried out with the technical staff of special schools for children with disabilities, in the state of Mato Grosso, between February and August 2017. McNemar's non-parametric test was performed to analyze the effect of the educational activity.

Results: 76 mid-level professionals participated, 62 (81.6\%) of whom were female and 66 (86.8\%) Child Development Assistants. Of the professionals, 35 (46.1\%) had never participated in training or an educational activity related to first aid. There was a significant increase in correct response to all the questions after the training $(p<.05)$.

Conclusion: the professionals of the technical staff presented insufficient prior knowledge regarding first aid and the training improved the safety of the students and contributed to the society in which they are included. The importance of the integration of nurses in the school environment was evidenced.
\end{abstract}

DESCRIPTORS: First aid; Training; Disabled people; Special education; Children.

\section{EVALUACIÓN DE ENTRENAMIENTO SOBRE PRIMEROS AUXILIOS PARA EL EQUIPO TECNICO DE ESCUELAS DE ENSENANZA ESPECIALIZADA}

\begin{abstract}
RESUMEN
Objetivo: analizar el efecto de actividad educativa sobre primeros auxilios en accidentes escolares.

Metodología: estudio casi experimental del tipo antes y después, realizado con el equipo técnico de escuelas de enseñanza especializada para personas con deficiencia, en el estado de Mato Grosso, Brasil, entre febrero y agosto de 2017. Fue realizado el test no paramétrico de McNemar para analizar el efecto de la actividad educativa.

Resultados: participaron 76 profesionales de nivel medio; 62 (81,6\%) eran del sexo femenino y 66 (86,8\%) eran Auxiliares de Desarrollo Infantil. De los profesionales, 35 (46,1\%) nunca participaron de entrenamientos o actividades educativa sobre primeros auxilios. Hubo significación estadística para el aumento de aciertos en todas las preguntas después del entrenamiento $(p<0,05)$.

Conclusión: los profesionales del equipo técnico presentaron conocimiento previo insuficiente sobre primeros auxilios y el entrenamiento mejoró la seguridad de los alumnos y contribuyó para la sociedad en que se encuentran inseridos. Se evidenció la importancia de la inserción del enfermero en el ambiente escolar.
\end{abstract}

DESCRIPTORES: Primeros auxilios; Capacitación; Personas con deficiencia; Educación especial; Niños. 
Segundo definição da Política Nacional de Redução da Morbimortalidade por Acidentes e Violências, acidente é "um evento não intencional e evitável, causador de lesões físicas e/ou emocionais no âmbito doméstico, ou nos outros ambientes sociais"(1). Estes eventos têm ocupado lugar de destaque entre as causas de morbimortalidade infantil e por isso são considerados problema de saúde pública em todo o mundo. Contudo, os dados disponíveis representam apenas minudência dos acidentes, pois muitos nem chegam a ser atendidos por serviços de saúde e pessoas presentes no momento do evento realizam os primeiros socorros ${ }^{(2-4)^{3}}$.

O ambiente escolar é um dos principais locais de ocorrência das injúrias não intencionais, e por isso o Ministério da Saúde e o Ministério da Educação estão trabalhando, através do Programa Saúde na Escola (PSE), para ampliar as ações de saúde nas escolas. Entre as metas previstas no âmbito do PSE se encontra a redução da morbimortalidade por acidentes, através de atividades de promoção de saúde nas escolas ${ }^{(5)}$.

Neste contexto estão as instituições de ensino especializado, que atendem à população infanto-juvenil com diferentes alterações de funcionalidade. Essas instituições objetivam complementar os serviços educacionais comuns, ao disponibilizar recursos e estratégias para a aprendizagem plena de alunos com deficiência ${ }^{(6)}$.

É preciso considerar que estudos sobre acidentes, realizados em escolas de educação especializada para pessoas com alterações de funcionalidade, evidenciaram maior risco para acidentes em crianças e adolescentes com deficiência no ambiente escolar ${ }^{(7-8)}$.

Outras casuísticas na Grécia, Estados Unidos e China também apontaram que crianças com deficiências físicas, cognitivas, sensitivas e múltiplas apresentam maiores riscos para as injúrias acidentais do que seus pares, sendo aquelas com deficiência múltipla e cognitiva apresentaram as lesões mais graves $^{(9-10)}$.

Dessa forma, ao considerar que a população infanto-juvenil com diferentes alterações de funcionalidade passa grande parte do período diurno dentro das instituições de ensino, e estão sujeitas à ocorrência de acidentes ou intercorrências neste ambiente, é extremamente necessário que todos os funcionários destas escolas estejam atentos às situações de risco e sejam capazes de instituir os primeiros socorros ${ }^{(11)}$.

Sabemos que não somente professores, mas toda a equipe técnica que trabalha nas escolas, deve estar preparada para promover a segurança dos alunos e reconhecer os sinais de perigo (11) em razão da Lei n 13.722 de 04 de Outubro de 2018, que torna obrigatória a capacitação em noções básicas de primeiros socorros de professores e funcionários de estabelecimentos de ensino públicos e privados de educação básica e de estabelecimentos de recreação infantil(12).

O conhecimento a respeito de primeiros socorros é essencial, uma vez que a abordagem correta em emergências pode salvar vidas e prevenir complicações adicionais ${ }^{(13)}$. Neste contexto, a educação em saúde é estratégia eficiente que pode ser realizada por enfermeiros, para capacitar a comunidade quanto aos primeiros socorros ${ }^{(14)}$. A educação em saúde promove a troca de conhecimento científico entre o profissional e os participantes, de forma a promover a autonomia e responsabilidade dos sujeitos quanto à prevenção de acidentes e atuação correta e eficiente frente a estes eventos ${ }^{(14)}$.

Este estudo tem como objetivo analisar o efeito de atividade educativa, sobre primeiros socorros em acidentes escolares, realizada para a equipe técnica de escolas de atendimento educacional especializado para pessoas com deficiência. 
Trata-se de estudo quase experimental do tipo antes e depois, com grupo único de comparação, realizado em escolas de atendimento educacional especializado de CuiabáMT e Várzea Grande-MT, durante o ano de 2017.

Cuiabá-MT e Várzea Grande-MT contam, respectivamente, com oito e duas escolas de atendimento educacional especializado para pessoas com deficiência. Todas as instituições receberam convite para participar do estudo, e seis aceitaram.

Foi disponibilizada uma data, dentro do quadro de horas dos funcionários, para participar da atividade educativa. Foram realizados seis treinamentos, um em cada instituição, e participaram do estudo 76 funcionários de nível técnico. A coleta de dados ocorreu entre os meses de fevereiro e agosto de 2017.

Neste estudo, considerou-se a equipe técnica destas instituições de ensino especializado: profissionais de nível médio e técnicos de escolaridade, que desenvolvem atividades em constante contato com as crianças, nos diferentes ambientes da escola: sala de aula, pátio, biblioteca, refeitório, banheiro, entre outros.

O instrumento de coleta de dados foi disponibilizado pelo Projeto Creche Segura (PCS) mediante autorização da autora. O Projeto Creche Segura é uma empresa de iniciativa privada, pioneira na formação de primeiros socorros no ambiente escolar ${ }^{(15)}$.

O questionário de coleta de dados é estruturado com oito perguntas fechadas e uma questão aberta, em linguagem não técnica, investigando conhecimento sobre como proceder diante das seguintes situações: 1- Queda infantil em ambiente escolar com trauma na cabeça; 2- Criança em momento de convulsão; 3- Trauma com perda de dente; 4-Bebê em situação de engasgo; 5- Criança maior ou adolescente em situação de engasgo; 6-Sequência de passos quando uma criança/adolescente não está respondendo e não apresenta pulso e respiração (situação de parada cardiorrespiratória); 7- Choque elétrico em ambiente escolar; 8- Queimadura por líquido quente (escaldadura); e Uma questão aberta perguntando qual o telefone do SAMU (Serviço de Atendimento Móvel de Urgência).

A atividade foi programada para uma duração média de $3 \mathrm{~h} 30 \mathrm{~min}$ a 4 horas. O Termo de Consentimento Livre e Esclarecido (TCLE) foi entregue em duas vias para todos os presentes, e lido em voz alta pela pesquisadora palestrante, explicando e esclarecendo dúvidas. Foi esclarecido que aqueles que não quisessem participar do estudo, não seriam impedidos de participar da atividade educativa, podendo permanecer na capacitação, não sendo preciso preencher e entregar o questionário de teste e o TCLE.

O pré-teste foi realizado com a entrega do questionário, sendo disponibilizado tempo de 25 minutos para resposta, sob supervisão das pesquisadoras. Após este período, os questionários foram recolhidos e iniciou-se a capacitação.

A primeira etapa ocorreu com a exposição do conteúdo teórico de forma expositiva e dialogada. Foram abordados os seguintes tópicos: prevenção de acidentes no ambiente escolar (quedas, queimaduras, intoxicações, choque elétrico, trauma devido a convulsões e acidentes com forças inanimadas e animadas), e primeiros socorros diante de acidentes (telefones dos serviços de emergência, como socorrer a criança vítima de queda, traumatismo craniano encefálico (TCE), convulsão, choque, trauma com avulsão dentária, queimadura, obstrução de vias aéreas e parada cardiorrespiratória). Os participantes tiveram espaço para expor dúvidas e compartilhar experiências. Esta atividade teve uma duração média de 90 minutos.

Posteriormente, seguiu-se a parte prática da capacitação e cada participante pôde fazer as seguintes atividades para memorização do conteúdo: manobra de desobstrução de via aérea no boneco pediátrico (manobra de Heimlich), demonstração da manobra na criança maior utilizando o colega, e procedimento de reanimação cardiopulmonar (RCP) nos manequins para simulação de RCP (pediátrico e adulto). Ỏ tempo médio para esta atividade foi de 40-50 minutos, e variou conforme o número de participantes em cada 
treinamento.

Seguidamente, foi realizado o pós-teste. Outro questionário foi entregue, contendo as mesmas perguntas do pré-teste, e foi disponibilizado um período de 25 minutos para resposta, sob as mesmas condições do primeiro teste.

Uma única equipe de pesquisadoras realizou o treinamento, com igual metodologia em todas as instituições participantes. O grupo era composto por uma professora de nível superior, doutoranda em saúde da criança e do adolescente, e duas acadêmicas de enfermagem, alunas de iniciação científica.

Os dados foram tabulados e analisados utilizando-se o programa Statistical Package for the Social Sciences (SPSS) versão 24. Foram utilizadas estatísticas descritivas e inferenciais para descrever as características da população de estudo e, para análise do efeito da atividade educativa sobre o conhecimento dos participantes a respeito dos primeiros socorros, foi realizado o teste não paramétrico de McNemar, por se tratar de amostra emparelhada e dados nominais.

O presente estudo foi aprovado pelo Comitê de Ética em pesquisa do Hospital Universitário Júlio Muller (HUJM), sob nº 1.689.650.

\section{RESULTADOS}

Participaram do estudo 76 profissionais de nível médio que trabalham em seis escolas de ensino especializado, sendo cinco em Cuiabá-MT e uma em Várzea Grande-MT. A idade mínima dos participantes foi de 20 anos e a máxima de 62 anos, com idade média de 36,62 , moda e mediana de 36 anos (Desvio padrão 10,25 anos).

A Tabela 1 evidencia a caracterização dos participantes quanto à instituição de ensino, sexo e ocupação. A escola com maior número de participantes foi a Escola $\mathrm{F}$ com $29(38,2 \%)$, o sexo feminino foi predominante entre os profissionais $(81,6 \%)$, o grupo etário mais prevalente foi entre $40-49$ anos $26(34,2 \%)$ e a principal ocupação de nível médio foi a de Auxiliares de Desenvolvimento Infantil (ADI) 66 (86,8\%). Apenas três escolas contam com o técnico de enfermagem na equipe técnica, duas escolas com um profissional em cada período e uma escola com um técnico em tempo integral, totalizando 6,6\% dos participantes (Tabela 1).

Tabela 1 - Perfil da equipe técnica das instituições de ensino especializado quanto à instituição, sexo, idade e ocupação. Cuiabá, MT, Brasil, 2017 (continua)

\begin{tabular}{lcc} 
ESCOLA & $\mathrm{n}$ & $\%$ \\
\hline Escola A & 2 & 2,6 \\
\hline Escola B & 20 & 26,3 \\
\hline Escola C & 11 & 14,5 \\
\hline Escola D & 7 & 9,2 \\
\hline Escola E & 7 & 9,2 \\
\hline Escola F & 29 & 38,2 \\
\hline Total & 76 & 100 \\
\hline SEXO & $\mathrm{n}$ & $\%$ \\
\hline
\end{tabular}




\begin{tabular}{lcc}
\hline Feminino & 62 & 81,6 \\
\hline Masculino & 14 & 18,4 \\
\hline TOTAL & 76 & 100 \\
\hline IDADE EM ANOS & $\mathrm{n}$ & $\%$ \\
\hline$<30$ & 25 & 32,9 \\
\hline $30-39$ & 18 & 23,7 \\
\hline $40-49$ & 26 & 34,2 \\
\hline$>50$ & 7 & 9,2 \\
\hline TOTAL & 76 & 100 \\
\hline PROFISSÃO & $\mathrm{n}$ & $\%$ \\
\hline Auxiliar de Desenvolvimento Infantil (ADI) & 66 & 86,8 \\
\hline Técnico de enfermagem & 5 & 6,6 \\
\hline Cozinheira & 5 & 6,6 \\
\hline TOTAL & 76 & 100
\end{tabular}

Um número significativo de profissionais nunca havia participado de um treinamento ou atividade educativa sobre primeiros socorros antes desta capacitação 35 (46,1\%). Entre os que haviam recebido treinamento anteriormente, mais da metade não passava por atualização há mais de dois anos (Tabela 2).

Tabela 2 - Capacitação da equipe técnica das instituições de ensino especializado quanto a prevenção de acidentes com crianças e primeiros socorros. Cuiabá, MT, Brasil, 2017

JÁ HAVIA PARTICIPADO DE UMA CAPACITAÇÃO SOBRE A TEMÁTICA

\begin{tabular}{lcc} 
JÁ HAVIA PARTICIPADO DE UMA CAPACITAÇÃO SOBRE A TEMÁTICA & $\mathrm{n}$ & $\%$ \\
\hline Sim & 41 & 53,9 \\
\hline Não & 35 & 46,1 \\
\hline TOTAL & 76 & 100 \\
\hline SE SIM, QUANTO TEMPO ATRÁS? & $\mathrm{n}$ & $\%$ \\
\hline Menos de 6 meses & 4 & 9,7 \\
\hline Entre 6 meses até 1 ano & 5 & 12,2 \\
\hline Entre 1 ano a 2 anos & 9 & 22 \\
\hline Entre 2 a 5 anos & 11 & 26,8 \\
\hline Acima de 5 anos & 12 & 29,3 \\
\hline TOTAL & 41 & 100
\end{tabular}

Quanto à capacitação prévia, 31(47,0\%) dos Auxiliares de Desenvolvimento Infantil e $4(80,0 \%)$ das cozinheiras não haviam realizado nenhuma capacitação a respeito de primeiros socorros e prevenção de acidentes (Tabela 3). 
Tabela 3 - Distribuição da equipe técnica das instituições de ensino especializado quanto à profissão e capacitação anterior sobre primeiros socorros. Cuiabá, MT, Brasil, 2017

Participou de capacitação sobre primeiros socorros

\begin{tabular}{lccc}
\hline Profissão & SIM $\mathbf{n}(\%)$ & NÃO $\mathbf{n}(\%)$ & TOTAL $\mathbf{n}(\%)$ \\
\hline ADI & $35(53)$ & $31(47)$ & $66(100)$ \\
\hline TÉC. DE ENFERMAGEM & $5(100)$ & - & $5(100)$ \\
\hline COZINHEIRA & $1(20)$ & $4(80)$ & $5(100)$ \\
\hline TOTAL & $41(53,9)$ & $35(46,1)$ & $76(100)$
\end{tabular}

teste estatístico McNemar mostrou que houve significância $(>0,05)$ no aumento de acerto das respostas em todas as questões investigadas. Na questão 3 , referente ao correto procedimento diante de trauma dentário, houve o maior número de erros antes da capacitação 72 (94,7\%), seguida da questão 2 referente à situação de convulsão e questões 5 e 6 referentes a engasgo e ressuscitação cardiopulmonar, respectivamente. Após a capacitação, as questões que tiveram maior número de acertos foram as questões referentes à queda infantil em ambiente escolar com trauma na cabeça e telefone do SAMU (Tabela 4).

Tabela 4 - Análise das respostas da equipe técnica das instituições de ensino especializado sobre primeiros socorros, antes e após a participação em atividade educativa. Cuiabá, MT, Brasil, 2017

\begin{tabular}{lccccc} 
Pergunta do questionário & \multicolumn{2}{c}{ ANTES n (\%) } & \multicolumn{2}{c}{ DEPOIS n (\%) } & Teste $^{\text {b }}$ \\
\cline { 2 - 6 } & CERTO & ERRADO & CERTO & ERRADO & \\
\hline Questão 1 & $57(75)$ & $19(25)$ & $72(94,7)$ & $4(5,3)$ & 0,001 \\
\hline Questão 2 & $17(22,4)$ & $59(77,6)$ & $59(77,6)$ & $17(22,4)$ & 0 \\
\hline Questão 3 & $4(5,3)$ & $72(94,7)$ & $59(77,6)$ & $17(22,4)$ & 0 \\
\hline Questão 4 & $39(51,3)$ & $37(48,7)$ & $63(82,9)$ & $13(17,1)$ & 0 \\
\hline Questão 5 & $34(44,7)$ & $42(55,3)$ & $63(82,9)$ & $13(17,1)$ & 0 \\
\hline Questão 6 & $34(44,7)$ & $42(55,3)$ & $70(92,1)$ & $6(7,9)$ & 0 \\
\hline Questão 7 & $50(65,8)$ & $26(34,2)$ & $70(92,1)$ & $6(7,9)$ & 0 \\
\hline Questão 8 & $36(47,4)$ & $40(52,6)$ & $70(92,1)$ & $6(7,9)$ & 0 \\
\hline Questão 9 & $43(56,6)$ & $33(43,4)$ & $72(94,7)$ & $4(5,3)$ & 0
\end{tabular}

${ }^{a}$ As temáticas das questões são: 1-Queda infantil com trauma na cabeça; 2- Convulsão; 3- Avulção dentária; 4-Bebê engasgado; 5- Criança maior/adolescente engasgado; 6- Parada cardiorespiratória; 7- Choque elétrico; 8- Queimadura por líquido quente (escaldadura). 9-telefone do SAMU.

${ }^{\mathrm{b}}$ Teste de McNemar 
Participaram do estudo 76 profissionais que trabalham em seis escolas de ensino especializado de Cuiabá-MT (cinco) e Várzea Grande-MT (uma). Houve significativa variação entre a idade dos participantes, contudo a faixa etária predominante (entre 40 a 49 anos) foi semelhante à de um estudo na Índia (Mangalore) ${ }^{(16)}$, e a média de idade foi semelhante à média encontrada em um estudo realizado em Portuga|(17).

A maior parte dos participantes é do sexo feminino, o que pode estar relacionado à função desempenhada: o ADI e o técnico de enfermagem exercem atividades relacionadas ao cuidar de pessoas. Achado semelhante foi evidenciado em estudo que avaliou o conhecimento de funcionários de uma escola(11) e outro realizado em Cuiabá-MT que avaliou o conhecimento de cuidadores de uma creche sobre prevenção de acidentes ${ }^{(18)}$.

Entre estes funcionários de nível médio e técnico, os Auxiliares do Desenvolvimento Infantil foram os profissionais em maior número. Este profissional atua junto às crianças e auxilia o professor no processo ensino-aprendizagem e no desenvolvimento de atividades recreativas, além de ser responsável pelos cuidados básicos de saúde das crianças (higiene, alimentação, repouso e bem estar), estando em constante contato com as crianças ${ }^{(19)}$.

Considerando o fato de o técnico de enfermagem ter papel fundamental na escola, sugere-se que este profissional continue realizando as atividades de capacitação dos professores e funcionários, de forma periódica e sob supervisão do enfermeiro como orienta o artigo 15 da Lei 7498/86, que dispõe sobre a regulamentação do exercício da Enfermagem e dá outras providências ${ }^{(5,20)}$.

Como não são todas as instituições que contam com uma equipe de enfermagem, sugerimos que a equipe de enfermagem da Estratégia de Saúde da Família (ESF), presente no bairro da unidade escolar, esteja desempenhando estas capacitações com regularidade, fortalecendo o vínculo entre a escola e a $\operatorname{ESF}^{(5,20)}$.

Outros estudos evidenciaram que os professores e funcionários de instituições educativas na maioria das vezes não recebem estas informações durante sua formação profissional e, mesmo quando recebem, muitos não se sentem totalmente capazes e seguros para realizarem as manobras diante de uma intercorrência, sendo essencial formação contínua e qualificada para aumentar a segurança(21-22).

Neste estudo, evidenciou-se número significativo de participantes que não haviam tido nenhuma capacitação prévia sobre prevenção de acidentes e primeiros socorros, sendo que este achado corrobora o de outras casuísticas ${ }^{(11-12,16)}$ e diverge de um estudo realizado em pré-escolas na Turquia(23).

Excluindo a presença do técnico de enfermagem na equipe, que em decorrência de sua formação foi capacitado para atuar nestas situações, chama a atenção a quantidade de ADI que nunca haviam recebido instruções anteriormente. Dado que o seu papel se desenvolve em íntimo contato com as crianças, pode ser o primeiro a evidenciar uma situação de risco ou acidente, sendo imprescindível que esteja capacitado para agir de forma correta, preservando a vida ou reduzindo os danos ${ }^{(13)}$.

Quanto ao conhecimento adquirido após a capacitação, houve aumento significante em todas as respostas corretas, corroborando os achados de outros estudos que evidenciaram significância no aumento do conhecimento após intervenção ${ }^{(11,13)}$.

Houve participantes que não sabiam como proceder corretamente diante de queda de nível alto. Evidencia-se a importância de divulgar o conhecimento pois, quando uma criança cai de nível alto, é fundamental que ela não seja movida, considerando a possibilidade de lesão da coluna, e o eixo cabeça-pescoço-corpo deve ser mantido (23). Acresce que crianças e adolescentes com alguma deficiência apresentam maior risco para TCE do que seus pares, principalmente quanto a criança apresenta paralisia cerebral, deficiência cognitiva ou deficiência múltipla ${ }^{(24)}$.

Crises convulsivas também surgem com maior frequência entre as crianças com 
deficiência. Contudo, no presente estudo, assim como no Sudão, foi evidenciado conhecimento insuficiente dos professores quanto ao correto manejo durante uma crise convulsiva: muitos acreditam que deve-se impedir os movimentos do corpo da criança, puxar a língua ou colocar objetos (como uma colher) na boca da criança, e não se preocupam em proteger a cabeça da criança e cronometrar o tempo da crise ${ }^{(25)}$.

Evidenciou-se insuficiente conhecimento prévio quanto ao meio de armazenamento do dente, em trauma com avulsão dentária. Resultado semelhante foi evidenciado em outro estudo(26) e em contraste com um estudo realizado em Matura (Índia)(27). O meio adequado para armazenamento do dente é o leite frio, em decorrência da sua composição, osmolaridade e $\mathrm{pH}$, podendo ficar imerso no leite por até 3 horas. Quando não se tem leite, pode-se utilizar soro fisiológico, contudo o mesmo tem eficiência por apenas 30 minutos. Já o armazenamento na saliva dentro da boca é contra indicada para este público pediátrico pelo risco de broncoaspiração do dente ${ }^{(27)}$.

Tal como o presente estudo, outros também evidenciaram conhecimento insuficiente dos participantes em relação à desobstrução de vias aéreas, parada cardiorrespiratória e choque elétrico, com significativo aumento após o treinamento ${ }^{(13,16,23)}$. Além do conteúdo teórico, a parte prática dos procedimentos de RPC e manobra de Heimlich são metodologias essenciais para o aprendizado e memorização do conteúdo, fundamentado segundo as orientações da American Heart Association ${ }^{(28-29)}$.

Quanto aos dados relativos à queimadura por escaldadura, cujo manejo imediato é resfriar a área queimada por no mínimo 15 minutos em água fria, limpa e corrente, foi outra casuística que também evidenciou equívoco do conhecimento dos professores ${ }^{(23)}$. Quando a área queimada não é previamente resfriada, a queimadura e os danos nos tecidos continuam a avançar para camadas mais profundas da pele, a hiperemia aumenta e pode formar flictenas ${ }^{(28)}$.

Um número significativo dos profissionais desconhecia o número correto do telefone de emergência, resultado semelhante ao de outros estudos ${ }^{(23,30)}$, evidenciando a necessidade das escolas divulgarem melhor estas informações de utilidade pública, e dos serviços de saúde se esforçarem por difundir para a população tanto o número de emergência, como as situações em que o serviço deve ser acionado.

O estudo apresentou como limitação a indisponibilidade de algumas instituições em liberar seus funcionários para participar do treinamento. Diante da importância destas atividades, sugere-se a inclusão de um período para capacitações desta natureza no planejamento do calendário escolar, de modo a que as mesmas ocorram, mantendo os funcionários sempre atualizados.

CONCLUSÃO

A classe de funcionários em maior número nos treinamentos foi a de Auxiliares de Desenvolvimento Infantil. Antes do treinamento, o conhecimento demonstrado sobre primeiros socorros diante dos principais acidentes escolares foi insuficiente, sendo que as questões com quase a totalidade de erros foram o correto manejo diante de trauma dentário, convulsão, desobstrução de vias aéreas e ressuscitação cardiopulmonar. Houve aumento, estatisticamente significativo, de acerto em todas as respostas após o treinamento, com destaque para as questões relativas à queda infantil em ambiente escolar com trauma na cabeça e ao número de telefone do SAMU.

Um número significativo de profissionais nunca havia participado de um treinamento ou atividade educativa sobre primeiros socorros antes deste estudo, e os resultados obtidos evidenciam a importância de capacitações para os profissionais que atuam nas escolas, corroborando com as propostas do Programa Saúde na Escola, com a Política Nacional de Redução de Política Nacional de Redução da Morbimortalidade por Acidentes e Violência 
e com a lei $n^{\circ} 13.722$, de 4 de outubro de 2018.

A capacitação destes profissionais de nível técnico, para atuarem frente a situações de acidentes, executando o correto procedimento de primeiros socorros, melhora a segurança dos alunos e contribui para a sociedade em que estão inseridos, uma vez que estarão capacitados para agir em outros contextos quando necessário.

Destaca-se a estratégia didática com metodologia prática que foi utilizada na capacitação, contribuindo para o resultado apresentado. A oportunidade para demonstração e execução das técnicas de primeiros socorros, frente a simulação de intercorrências, permitiu maior fixação do conteúdo teórico e relação do conhecimento científico com situações reais compartilhadas entre os participantes.

O presente estudo realça importantes contribuições para a enfermagem, evidenciando a importância da inserção destes profissionais junto a diferentes contextos na comunidade, por exemplo em escolas, desenvolvendo atividades de educação em saúde e capacitando a população quanto à temática.

\section{REFERÊNCIAS}

1. Brasil. Política nacional de redução da morbimortalidade por acidentes e violências: Portaria MS/GM n.737 de 16/05/01 publicada no DOU n.96 seção 1e de 18/05/01. Brasília: Ministério da Saúde; 2003.

2. Barcelos RS, Santos IS, Matijasevich A, Barros AJD, Barros FC, França GVA, et al. Acidentes por quedas, cortes e queimaduras em crianças de 0-4 anos: coorte de nascimentos de Pelotas, Rio Grande do Sul, Brasil, 2004. Cad. Saúde Pública [Internet]. 2017 [acesso em 04 jan 2019]; 33(2). Disponível em: http://dx.doi.org/10.1590/0102-311x00139115.

3. World Health Organisation. World report on child injury prevention. Geneva, Switz. 2008; 1-212.

4. Sengoelge M, Hasselberg M, Laflamme L. Child home injury mortality in Europe: A 16-country analysis. Eur J Public Health. Internet]. 2011 [acesso em 04 jan 2019]; 21(2). Disponível em: http://dx.doi. org/10.1093/eurpub/ckq047.

5. Brasil. Ministério da Saúde. Caderno de atenção básica no 24: saúde na escola. Brasília: Editora MS; 2009. 96 p.

6. Conselho Nacional de Educação. Resolução n. 4, de 2 outubro 2009. Institui Diretrizes Operacionais para o Atendimento Educacional Especializado na Educação Básica, modalidade Educação Especial. Brasilia: CNE; 2009.

7. Ramirez M, Fillmore E, Chen A, Peek-Asa C. A comparison of school injuries between children with and without disabilities. Acad Pediatr. [Internet]. 2010 [acesso em 04 jan 2019]; 10(5). Disponível em: https:// doi.org/10.1016/j.acap.2010.06.003.

8. Ramirez M, Fillmore E, Chen A, Peek-Asa C. A comparison of school injuries between children with and without disabilities. Acad Pediatr. [Internet]. 2010 [acesso em 04 jan 2019]; 10(5). Disponível em: https:// doi.org/10.1016/j.acap.2010.06.003.

9. Sinclair SA, Xiang H. Injuries among US children with different types of disabilities. Am J Public Health. [Internet]. 2008 [acesso em 04 jan 2019]; 98(8). Disponível em: https://doi.org/10.2105/ AJPH.2006.097097.

10. Xiang $\mathrm{H}$, Wheeler KK, Stallones L. Disability status: A risk factor in injury epidemiologic research. Ann Epidemiol [Internet]. 2014 [acesso em 04 jan 2019]; 24(1). Disponível em: https://doi.org/10.1016/j. annepidem.2013.10.014.

11. Calandrim LF, Santos AB dos, Oliveira LR de, Massaro LG, Vedovato CA, 
Boaventura AP. First aid at school: teacher and staff training. Rev RENE [Internet]. 2017 [acesso em 04 jan 2019];18(3):292-9. Disponível em: http://dx.doi.org/10.15253/2175-6783.2017000300002.

12. Brasil. Lei n. 13.722, de 4 de outubro de 2018. Torna obrigatória a capacitação em noções básicas de primeiros socorros de professores e funcionários de estabelecimentos de ensino públicos e privados de educação básica e de estabelecimentos de recreação infantil. Diário Oficial da República Federativa do Brasil, Brasília, 4 out. 2018. Seção 1:1.

13. Li F, Sheng $X$, Zhang J, Jiang F, Shen X. Effects of pediatric first aid training on preschool teachers: a longitudinal cohort study in China. BMC Pediatr [Internet]. 2014 [acesso em 04 jan 2019]; 14(1). Disponível em: https://doi.org/10.1186/1471-2431-14-209.

14. Galdino Neto NM, Sá GG de M, Vasconcelos EMR de, Silva TM da, Santos AMR dos, Carvalho KM de. Intervenções de educação em saúde sobre primeiros socorros para leigos no brasil: revisão integrativa. Ciênc. cuid. saúde. [Internet]. 2017 [acesso em 04 jan 2019]; 16(4). Disponível em: http://dx.doi. org/10.4025/cienccuidsaude.v16i4.38305.

15. Projeto Creche Segura [Internet]. 2018 [acesso em 17 mar 2018]. Disponível em: http://www. crechesegura.com.br/.

16. Joseph N, Narayanan T, Zakaria S bin, Nair AV, Subramanian AM, Gopakumar KG. Awareness, attitudes and practices of first aid among school teachers in Mangalore, south India. J Prim Health Care [Internet]. 2015[acesso em 04 jan 2019];7(4):274-81. Disponível em: https://www.ncbi.nlm.nih.gov/ pubmed/26668832.

17. Esteves D, Pinheiro P, Brás R, O'Hara K, Rodrigues R. Avaliação do conhecimento dos professores de educação física para reagirem a situações de emergência. Motricidade [Internet]. 2015 [acesso em 04 jan 2019];11(1). Disponível em: https://doi.org/10.6063/motricidade.3125.

18. Costa SNG da C, Silva JMM da, Freitas BHBM, Reis AFC. Acidentes infantis: conhecimento e percepção de educadoras de creches. Rev enferm UFPE online [Internet]. 2017[acesso em 04 jan 2019]; 11(10). Disponível em: https://periodicos.ufpe.br/revistas/revistaenfermagem/article/ download/69696/24314.

19. Paulino VBR, Côco V. Vozes do trabalho docente na educação infantil. SciELO em Perspectiva: Humanas [Internet]. 2016 [acesso em 26 abril 2019]; 24(92). Disponível em: http://humanas.blog.scielo. org/blog/2016/08/29/vozes-do-trabalho-docente-na-educacao-infantil/.

20. Brasil. Lei n. 7.498, de 25 de junho de 1986. Dispõe sobre a Regulamentação do Exercício da Enfermagem e dá outras providências. Diário Oficial da República Federativa do Brasil, Brasília, 26 jun. 1986. Seção 1:1.

21. Silva LGS, Costa JB da, Furtado LGS, Tavares JB, Costa JLD. Primeiros socorros e prevenção de acidentes no ambiente escolar : os professores não em situações que da criança, gerando risco. Enferm Foco [Internet]. 2017 [acesso em 04 jan 2019]; 8(3). Disponível em: http://biblioteca.cofen.gov.br/wpcontent/uploads/2018/03/Primeiros-socorros-e-preven\%C3\%A7\%C3\%A3o-de-acidentes-no-ambienteescolar.pdf.

22. Carmo H de O, Souza RC de A, Araújo CL de O, Francisco AG. Atitudes dos docentes de educação infantil em situação de acidente escolar. Rev. enferm. Cent.-Oeste Min. [Internet]. 2017[acesso em 04 jan 2019];7(e1457). Disponível em: http://dx.doi.org/10.19175/recom.v7i0.1457.

23. Sönmez Y, Uskun E, Pehlivan A. Knowledge levels of pre-school teachers related with basic first-aid practices, Isparta sample. Turk Pediatr Ars [Internet]. 2014 [acesso em 04 jan 2019]; 49(3). Disponível em: http://dx.doi.org/10.5152/tpa.2014.1581.

24. Limbos MAP, Ramirez M, Park LS, Peek-asa C, Kraus JF. Injuries to the Head Among Children Enrolled in Special Education. Arch Pediatr Adolesc Med. [Internet]. 2004 [acesso em 04 jan 2019]; 158(11):105761. Disponível em: http://dx.doi.org/10.1001/archpedi.158.11.1057.

25. Elhassan MA, Alemairy AA, Amara ZM, Hamadelneel AA, Mohamed AH, Elaimeri AA. Epilepsy: 
Knowledge, Attitude, and Practice Among Secondary School Teachers in Khartoum State. Neurol ther. [Internet]. 2017 [acesso em 04 jan 2019]; 6(2). Disponível em: http://dx.doi.org/10.1007/s40120-017-00837.

26. Attarzadeh H, Kebriaei F, Sadri L, Foroughi E, Taghian M. Knowledge and Attitudes of Elementary Schoolteachers on Dental Trauma and its Management in Yazd, Iran. J Dent [Internet]. 2017 [acesso em 04 jan 2019]; 18(3):212-8. Disponível em: https://www.ncbi.nlm.nih.gov/pubmed/29034277.

27. Singh M, Yadav P, Ingle N, Kaur N. Evaluation of knowledge and attitude of school teachers about emergency management of traumatic dental injury. J Int Soc Prev Community Dent [Internet]. 2015 [acesso em 04 jan 2019]; 5(2). Disponível em: http://dx.doi.org/10.4103/2231-0762.155735.

28. Atkins DL, Berger S, Duff JP, Gonzales JC, Hunt EA, Joyner BL, et al. Part 11: pediatric basic life support and cardiopulmonary resuscitation quality: 2015. American Heart Association guidelines update for cardiopulmonary resuscitation and emergency cardiovascular care. Circulation[Internet]. 2015 [acesso em 04 jan 2019]; 132(18 Suppl 2):S519-25. Disponível em: https://doi.org/10.1161/ CIR.0000000000000265.

29. Markenson D, Ferguson JD, Chameides L, Cassan P, Chung KL, Epstein J, et al. Part 17: first aid: 2010 American Heart Association and American Red Cross Guidelines for First Aid. Circulation [Internet]. 2010 [acesso em 04 jan 2019]; 122(18 Suppl 3) S934-45. Disponível em: https://doi.org/10.1161/ CIRCULATIONAHA.110.971150.

30. Slabe D, Fink R, Dolenc E, Kvas A. Knowledge of health principles among professionals in Slovenian kindergartens. Zdrav Var [Internet]. 2016 [acesso em 04 jan 2019]; 55(3):185-194. Disponível em: http:// dx.doi.org/10.1515/sjph-2016-0024.

Recebido: $13 / 07 / 2018$

Finalizado: 08/05/2019

Autor Correspondente:

Jackeline Gonçalves Brito

Universidade Federal de Mato Grosso

Av. Fernando Corrêa da Costa, 2367 - 78060-900 - Cuiabá, MT, Brasil

E-mail: jackeline_brito@hotmail.com

Contribuição dos autores:

Contribuições substanciais para a concepção ou desenho do estudo; ou a aquisição, análise ou interpretação de dados do estudo - JGB, IMS, CBG, APSJMF

Elaboração e revisão crítica do conteúdo intelectual do estudo - JGB, IMS, CBG, APSJMF

Aprovação da versão final do estudo a ser publicado - JGB, IMS, CBG, APSJMF 